\title{
ARTICLES
}

\section{Modified WKB approximation}

\author{
J. B. Bronzan* \\ CERN, CH-1211 Geneva 23, Switzerland \\ and Department of Physics and Astronomy, Rutgers University, Piscataway, New Jersey 08855-0849 \\ (Received 3 March 1995; revised manuscript received 23 August 1995)
}

\begin{abstract}
In the WKB approximation the $\nabla^{2} S$ term in Schrödinger's equation is subordinate to the $|\nabla S|^{2}$ term. Here we study a modified WKB approximation in which the $\nabla^{2} S$ term dominates (after a guess for $S$ is supplied). Our approximation produces only the nodeless ground-state wave function, but unlike the WKB approximation it can be applied straightforwardly to problems having many degrees of freedom. As a test, we apply the method to potential problems, including the hydrogen and helium atoms, and to $\phi^{4}$ field theory. We show that good numerical results for the bound-state energy can be obtained even when the initial guess for $S$ is manifestly inaccurate. Our method supplies the wave function as well as the energy. [S1050-2947(96)05007-X]
\end{abstract}

PACS number(s): 03.65.Ge, 02.30.Mv, 03.65.Db

\section{INTRODUCTION}

In quantum mechanics the wave function is sometimes written in the form

$$
\psi(\mathbf{r})=\exp S(\mathbf{r})
$$

Schrödinger's equation then takes the form

$$
\nabla^{2} S+|\boldsymbol{\nabla} S|^{2}=\frac{2 m}{\hbar^{2}}[V(\mathbf{r})-E]
$$

Equation (1.2) is the quantum Hamilton-Jacobi equation. Here it has been deduced from Schrödinger's equation. Others have taken it as a basic postulate from which quantum mechanics is constructed [1,2]. Leacock and Padgett have introduced angle-action variables and based an effective scheme for computing energy eigenvalues on the HamiltonJacobi equation [1].

The WKB approximation ensues when the term $\nabla^{2} S$ term in Eq. (1.2) is dropped in leading order and later incorporated as a correction [3]. It is commonly understood that this step is justified in the semiclassical regime where the gradient of the de Broglie wavelength, $\nabla \lambda$, has a magnitude much smaller than one, and the wave function oscillates many times over distances that characterize the variation of the potential.

In this paper we consider the alternate ordering of terms where the term $|\nabla S|^{2}$ is dropped in leading order and later treated as a correction. This is a modified WKB approximation. Here we develop the modified WKB approximation for nodeless wave functions. For a particle moving in a potential, such a wave function describes a bound state, and in a bosonic field theory it describes the ground state. Thus the modified WKB approximation, as developed here, augments the WKB approximation in an important case where the

\footnotetext{
*Electronic address: bronzan@physics.rutgers.edu
}

WKB approximation can be expected to be numerically inadequate. An additional contrast with the WKB approximation is that the modified WKB approximation can be applied straightforwardly to problems having many degrees of freedom.

The modified WKB approximation is not as straightforward as the WKB approximation because it is not generally true that $\left|\nabla^{2} S\right| \gg|\nabla S|^{2}$ for bound-state wave functions. To see this explicitly, assume the potential vanishes when $r>a$. Then for $r>a$,

$$
S=S_{0}-K r-\frac{D-1}{2} \ln \left(r / r_{0}\right), \quad K \equiv\left(-\frac{2 m E}{\hbar^{2}}\right)^{1 / 2},
$$

where motion is in $D$ spatial dimensions. Comparing the gradient and Laplacian applied to this expression, we find that the modified WKB approximation is justified only when $D=1$ and $K=0$ (or at least $K \sim 0$.) This is the case of a weakly bound particle moving in one dimension. (Recall that in one dimension there is always one bound state in an attractive potential, no matter how weak the potential.) This version of the modified WKB approximation has been developed [4]. One obtains an expression for the energy of the bound state as a series of integrals over powers of the potential. The relative size of the $n$th term is proportional to the $n$th power of the parameter $V_{0} m a^{2} / \hbar^{2}$, where $V_{0}$ is the strength of the potential and $a$ its range. We see here an expected contrast with the WKB approximation: a series of decreasing powers of Planck's constant. Despite this, if the potential is such as to make the parameter small, the approximation succeeds.

The straightforward case just described is quite different from the modified WKB approximation considered in this paper, where we treat the general case $D \neq 1, K \neq 0$. We evade the "no-go", conclusion, above, by supplying an initial guess for $S$. Thus we write 


$$
S(\mathbf{r})=F(\mathbf{r})+T(\mathbf{r}),
$$

where the seed $F$ has the asymptotic form (1.3), and is chosen to be an initial guess for $S$. The correction $T$ is now determined by our version of the quantum Hamilton-Jacobi equation:

$$
\begin{aligned}
\nabla^{2} T_{n}+2 \boldsymbol{\nabla} F \cdot \boldsymbol{\nabla} T_{n}= & \frac{2 m}{\hbar^{2}}\left[V(\mathbf{r})-E_{n}\right]-\nabla^{2} F-|\boldsymbol{\nabla} F|^{2} \\
& -\left|\boldsymbol{\nabla} T_{n-1}\right|^{2}, \quad T_{-1}=0 .
\end{aligned}
$$

The index $n=0,1, \ldots$ labels the successive approximations to $T$ and hence $S$. The initial approximation ignores the gradient of $T$; the next approximation uses the initial approximation for the gradient, and so forth. Note that for each $n$ we obtain a different approximation to the bound-state energy $E_{n}$. Although Eq. (1.5) is written for a problem with one degree of freedom, the same equation holds for many degrees of freedom provided the differential operators are taken to operate in a space of appropriate dimension.

Of course, ignoring the gradient of $T$ in Eq. (1.5) is different from ignoring the gradient of $S$ in Eq. (1.2) because much of the gradient of $S$ is supplied by the term $|\nabla F|^{2}$. Nevertheless, the modified WKB approximation follows the opposite strategy to that of the WKB approximation in treating terms in the quantum Hamilton-Jacobi equation. Here the Laplacian term is treated exactly and the gradient term through successive approximations.

In Sec. II we demonstrate that this sequence of approximations is formally convergent provided $F$ is well chosen. It is useful to state what we find. Assume that our seed differs from $S$ by a function scaled by a small parameter $\epsilon$ :

$$
F(\mathbf{r})=S(\mathbf{r})-\epsilon S_{1}(\mathbf{r}) .
$$

It follows that the exact $T$ is $\epsilon S_{1}$. In Sec. II we show that

$$
E_{n}=E+\epsilon^{n+2} A_{n}, \quad T_{n}=\epsilon S_{1}+\epsilon^{n+2} R_{n} .
$$

Here $E$ is the true bound-state energy, and the factors $A_{n}$ and $R_{n}$ are finite at $\epsilon=0$. The convergence is formal because we have not given estimates for the $n$ dependence of $A_{n}$ and $R_{n}$. If these factors increase with $n$ faster than an exponential, the sequence does not converge, but is instead asymptotic.

The convergence estimates of Eq. (1.7) depend on the fact that the term $T_{n-1}$ appears quadratically on the right-hand side of Eq. (1.5). When $F$ differs little from $S, T$ is small, and $\left|\nabla T_{n-1}\right|^{2}$ is doubly small. In Sec. III we will show how the modified WKB approximation works in an example where several approximants are evaluated. There we verify that the accuracy of $E_{n}$, at given $n$, depends on the choice of $F$. However, the same example will show that good results can be obtained, even with a very poor choice of $F$, when $n$ is only 2 .

We again emphasize the difference between what is done in Ref. [4] and what we do here. This is not a weak potential approximation, nor is it an expansion in inverse powers of $\hbar$. Here we are not limited to cases where the wave function changes slowly over the distance characterizing the variation of the potential. All of these limitations, present in Ref. [4], are lifted when the seed $F$ is introduced. The present modified WKB approximation is an approximation in which an initial guess $F$ is systematically improved by our version of the quantum Hamilton-Jacobi equation, Eq. (1.5). The sequence of approximations is formally convergent. To carry out the approximation we require a reasonable seed $F$ for which the integrals and Green functions we encounter can be computed. Choosing $F$ is equivalent to choosing a trial ground-state wave function, and $E_{0}$ is the corresponding variational estimate of the ground-state energy. These entities are required to initiate the sequence of modified WKB approximants, but they stand apart from those approximants. The first modified WKB approximant to $S$ is $F+T_{0}$, and the first modified WKB approximant to the ground-state energy is $E_{1}$.

The wave function arising from our construction is nodeless. The reason is that if $\psi$ vanishes on the surface $f(\mathbf{r})=0$, there will be a term $\ln f(\mathbf{r})$ in $S$. In the neighborhood of the node, this logarithm dominates $S$, and we must explicitly incorporate it into $F$ if we obtain a wave function with a node. Note that we must specify the surface $f=0$, which is known only under special circumstances. We do not consider such cases here; our $F$ 's will be smooth, and we therefore limit ourselves to nodeless wave functions. This means that the energies $E_{n}$ are approximations to the groundstate energy $E$. In Ref. [1] the solution of the quantum Hamilton-Jacobi equation for bound states with nodes is discussed, but we do not do so in this paper.

Like the WKB approximation, the modified WKB approximation is nonperturbative; it does not require the presence of a small parameter in the Hamiltonian. Its major limitation is that it is restricted to the ground-state wave function. But the modified WKB approximation has this important advantage: Equation (1.5) for $T_{n}$ is linear. We will see that it can be solved readily in many cases of interest, including particles moving in three dimensions in nonspherical potentials, and many-body problems like bosonic lattice field theory. In the latter problem the method can be extended to study some of the vacuum state correlation functions that are of central importance in field theory. All these possibilities are closed to the WKB approximation, which is generally unmanageable except for a particle whose motion effectively reduces to one dimension.

In Sec. II we present a theoretical development of the modified WKB approximation. We include the solution of the dynamical equation (1.5), the convergence of the sequence of approximations, and several other matters.

In following sections the modified WKB approximation is applied to a number of problems to show that it works in increasingly complex situations. In Sec. III we study a spherically symmetric square well whose ground state is known by elementary methods. It is for this case that we study the dependence on the seed $F$ and the accuracy attainable with higher approximants. There we show that even with the worst $F$ of the four we study, we obtain an energy that differs from the exact energy by $0.31 \%$ when $n=2$. In Sec. III we also develop Green functions required for the application of Eq. (1.5) to potential problems where the potential is nonspherical. In Sec. IV we study long-range potentials, with the hydrogen atom as a particularly simple subcase. We finish by applying the modified WKB 
approximation to many-body problems: $\phi^{4}$ field theory in Sec. V, and the helium atom in Sec. VI. Conclusions are presented in Sec. VII.

\section{MODIFIED WKB EQUATIONS}

The energies in Eq. (1.5) are determined by a general requirement. Consider the surface integral

$$
\oint_{S} d \mathbf{A} \cdot\left[e^{2 F} \boldsymbol{\nabla} T_{n}\right]=\int_{V} d V \boldsymbol{\nabla} \cdot\left[e^{2 F} \boldsymbol{\nabla} T_{n}\right]
$$

As $S$ is expanded to infinity, the surface integral decreases to zero because of the exponential fall of the factor $e^{2 F}$. Using Eq. (1.5) we obtain the eigenvalue equation determining $E_{n}$ :

$0=\int d V e^{2 F}\left\{\frac{2 m}{\hbar^{2}}\left[V(\mathbf{r})-E_{n}\right]-\nabla^{2} F-|\nabla F|^{2}-\left|\nabla T_{n-1}\right|^{2}\right\}$.

We can now assemble the equations to derive the convergence results (1.7). The true ground-state energy $E$ is determined by Eq. (2.2) with the replacements $E_{n} \rightarrow E$, $T_{n-1} \rightarrow T$. Subtracting equations,

$$
\begin{gathered}
0=\int d V e^{2 F}\left[\frac{2 m}{\hbar^{2}}\left(E_{n}-E\right)+\left|\nabla T_{n-1}\right|^{2}-|\nabla T|^{2}\right], \\
E_{n}=E+\frac{\hbar^{2}}{2 m} \frac{\int d V e^{2 F}\left[|\nabla T|^{2}-\left|\nabla T_{n-1}\right|^{2}\right]}{\int d V e^{2 F}} .
\end{gathered}
$$

We now express the $n$ th-order factors in terms of their limiting values and a deviation:

$$
E_{n}=E+a_{n}, \quad T_{n}=-\epsilon S_{1}+r_{n} .
$$

Then Eq. (2.3) becomes

$$
\begin{gathered}
a_{0}=\epsilon^{2} \frac{\hbar^{2}}{2 m} \frac{\int d V e^{2 F}\left|\nabla S_{1}\right|^{2}}{\int d V e^{2 F}}, \\
a_{n}=\frac{\hbar^{2}}{2 m} \frac{\int d V e^{2 F}\left[2 \epsilon \boldsymbol{\nabla} S_{1} \cdot \nabla r_{n-1}-\left|\nabla r_{n-1}\right|^{2}\right]}{\int d V e^{2 F}} \quad(n \geqslant 1) .
\end{gathered}
$$

We next use Eq. (1.5) and the analogous equation for $T=\epsilon S_{1}$. Subtracting the equations

$$
\begin{gathered}
\nabla^{2} r_{0}+2 \boldsymbol{\nabla} F \cdot \boldsymbol{\nabla} r_{0}=-\frac{2 m}{\hbar^{2}} a_{0}+\epsilon^{2}\left|\nabla S_{1}\right|^{2} \\
\nabla^{2} r_{n}+2 \boldsymbol{\nabla} F \cdot \boldsymbol{\nabla} r_{n}=-\frac{2 m}{\hbar^{2}} a_{n}+2 \epsilon \boldsymbol{\nabla} S_{1} \cdot \boldsymbol{\nabla} r_{n-1}-\left|\nabla r_{n-1}\right|^{2}
\end{gathered}
$$

$$
(n \geqslant 1)
$$

It follows that Eq. (1.7) holds for $n=0$. Furthermore, if (1.7) holds for $n$, then from Eqs. (2.5), (2.6) we see that it holds for $n+1$, and the result is established by induction.

Energy $E_{n}$ requires $T_{n-1}$ for its computation. This information can be used to find a better estimate of the state energy from the expectation

$$
\mathcal{E}_{n}=\frac{\langle\psi|H| \psi\rangle}{\langle\psi \mid \psi\rangle}, \quad \psi=\exp \left(F+T_{n-1}\right) .
$$

The error in $\psi$ is $O\left(\epsilon^{n+1}\right)$; variational argument [5] gives

$$
\mathcal{E}_{n}-E=O\left(\epsilon^{2 n+2}\right), \quad \frac{\mathcal{E}_{n}-E}{\left|E_{n}-E\right|}=O\left(\epsilon^{n}\right) .
$$

Equation (1.5) may be solved using the Green function satisfying

$$
L G\left(\mathbf{r}, \mathbf{r}^{\prime}\right)=\delta\left(\mathbf{r}-\mathbf{r}^{\prime}\right), \quad L=\nabla^{2}+2(\boldsymbol{\nabla} F) \cdot \nabla .
$$

Then

$$
\begin{aligned}
T_{n}(\mathbf{r})= & \int d V^{\prime} G\left(\mathbf{r}, \mathbf{r}^{\prime}\right)\left\{\frac{2 m}{\hbar^{2}}\left[V-E_{n}\right]-\nabla^{2} F-|\nabla F|^{2}\right. \\
& \left.-\left|\nabla T_{n-1}\right|^{2}\right\}_{\mathbf{r}^{\prime}}
\end{aligned}
$$

It is illuminating to expand the Green function in terms of the orthonormal eigenfunctions of $L$, because the argument leads to the eigenvalue equation (2.2) in a different way.

$$
L \phi_{k}=\mu_{k} \phi_{k}, \quad \int d V e^{2 F} \phi_{k 1} \phi_{k 2}=\delta_{k 1, k 2} \text {. }
$$

Then

$$
G\left(\mathbf{r}, \mathbf{r}^{\prime}\right)=\sum_{k} \frac{1}{\mu_{k}} \phi_{k}(\mathbf{r}) \phi_{k}\left(\mathbf{r}^{\prime}\right) e^{2 F\left(\mathbf{r}^{\prime}\right)}
$$

But there is a problem: $L$ annihilates a constant function, so there is a normalized eigenfunction of $L$ with eigenvalue zero:

$$
\phi_{0}(\mathbf{r})=N, \quad N=\left[\int d V e^{2 F}\right]^{-1 / 2}, \quad \mu_{0}=0 .
$$

The contribution of this zero mode to $G$ is infinite. Nonetheless, $T_{n}$ in Eq. (2.10) is finite if the projection of the driving term onto the zero mode vanishes. The condition for that is the eigenvalue equation (2.2).

The eigenvalue equation for $E_{1}$ can be simplified. We need the integral 


$$
\begin{aligned}
-\int d V e^{2 F}\left|\boldsymbol{\nabla} T_{0}\right|^{2}= & -\int d V \boldsymbol{\nabla} \cdot\left[e^{2 F} T_{0} \boldsymbol{\nabla} T_{0}\right] \\
& +\int d V T_{0} \boldsymbol{\nabla} \cdot\left[e^{2 F} \boldsymbol{\nabla} T_{0}\right] .
\end{aligned}
$$

The first integral on the right vanishes by the divergence theorem, and the second may be transformed using Eq. (1.5).

$$
\begin{aligned}
-\int d V e^{2 F}\left|\boldsymbol{\nabla} T_{0}\right|^{2} & =\int d V e^{2 F} T_{0} D \\
& =\int d V d V^{\prime} e^{2 F(\mathbf{r})} D(\mathbf{r}) D\left(\mathbf{r}^{\prime}\right) G\left(\mathbf{r}, \mathbf{r}^{\prime}\right)
\end{aligned}
$$

where

$$
D \equiv \frac{2 m}{\hbar^{2}}\left[V-E_{0}\right]-\nabla^{2} F-|\nabla F|^{2}
$$

The quantization condition for $E_{1}$ reads

$$
\begin{aligned}
0= & \int d V e^{2 F}\left[D+\frac{2 m}{\hbar^{2}}\left(E_{0}-E_{1}\right)\right] \\
& +\int d V d V^{\prime} e^{2 F(\mathbf{r})} D(\mathbf{r}) D\left(\mathbf{r}^{\prime}\right) G\left(\mathbf{r}, \mathbf{r}^{\prime}\right), \\
E_{1}=E_{0} & +\frac{\hbar^{2}}{2 m} \frac{\int d V d V^{\prime} e^{2 F(\mathbf{r})} D(\mathbf{r}) D\left(\mathbf{r}^{\prime}\right) G\left(\mathbf{r}, \mathbf{r}^{\prime}\right)}{\int d V e^{2 F}} .
\end{aligned}
$$

When $V(\mathbf{r})$ depends on a single coordinate, all differential equations involved in constructing the Green function can be solved. Consider the case where $V, F$, and $T$ depend only on the radial coordinate, as happens in Sec. III. Then the Green function satisfies the ordinary differential equation

$$
L g\left(r, r^{\prime}\right)=\frac{1}{r^{\prime 2}} \delta\left(r-r^{\prime}\right), \quad L=\frac{d^{2}}{d r^{2}}+2\left(\frac{1}{r}+\frac{d F}{d r}\right) \frac{d}{d r}
$$

Rather than expand in eigenfunctions, we construct $g$ from the two solutions of $L R=0$, which are known.

$$
R^{+}(r)=1, \quad R^{-}(r)=\int_{r_{0}}^{r} \frac{d r^{\prime} e^{-2 F\left(r^{\prime}\right)}}{r^{\prime 2}} .
$$

The Green function is

$$
\begin{aligned}
g\left(r, r^{\prime}\right) & =R^{+}\left(r_{>}\right) R^{-}\left(r_{<}\right) / r^{\prime 2} W\left(r^{\prime}\right) \\
& =-R^{+}\left(r_{>}\right) R^{-}\left(r_{<}\right) e^{2 F\left(r^{\prime}\right)}
\end{aligned}
$$

where $W$ is the Wronskian

$$
W\left(R^{+}, R^{-}\right) \equiv \frac{d R^{+}}{d r} R^{-}-R^{+} \frac{d R^{-}}{d r}=-\frac{e^{-2 F(r)}}{r^{2}} .
$$

Now

$$
\begin{aligned}
T_{n}(r)= & \int_{0}^{\infty} d r^{\prime} r^{\prime 2} g\left(r, r^{\prime}\right)\left\{\frac{2 m}{\hbar^{2}}\left[V-E_{n}\right]-F^{\prime \prime}-\frac{2}{r^{\prime}} F^{\prime}\right. \\
& \left.-\left(F^{\prime}\right)^{2}-\left(T_{n-1}^{\prime}\right)^{2}\right\}_{r^{\prime}}
\end{aligned}
$$

The eigenvalue equation emerges here, not through a zero mode, but through the bad asymptotic behavior of $R^{-}$. Near $r=0, R^{-}(r) \sim e^{-2 F(0)} / r$, and

$$
\begin{aligned}
T_{n}(r) \sim & \frac{e^{-2 F(0)}}{r} \int_{0}^{\infty} d r^{\prime} r^{\prime 2} e^{2 F\left(r^{\prime}\right)}\left\{\frac{2 m}{\hbar^{2}}\left[V-E_{n}\right]-F^{\prime \prime}-\frac{2}{r^{\prime}} F^{\prime}\right. \\
& \left.-\left(F^{\prime}\right)^{2}-\left(T_{n-1}^{\prime}\right)^{2}\right\}_{r^{\prime}}
\end{aligned}
$$

To keep $T_{n}$ finite at $r=0$, the eigenvalue integral must vanish; when it does, $T_{n}(0)=0$.

\section{SPHERICAL SQUARE WELL POTENTIAL}

We begin our study of the modified WKB approximation by applying it to the case of the spherically symmetric potential

$$
V(r)= \begin{cases}-V_{0} & (r<a) \\ 0 & (r>a)\end{cases}
$$

The radial Schrödinger equation for $s$-wave bound states can be solved, and the energies are determined by the equation [6]

$$
\begin{gathered}
k a \cot k a=-K a, \quad k a=\left(\frac{2 m a^{2}}{\hbar^{2}} V_{0}-(K a)^{2}\right)^{1 / 2}, \\
E=-\frac{\hbar^{2} K^{2}}{2 m} .
\end{gathered}
$$

There is no bound state unless $V_{0}$ exceeds the threshold strength

$$
\left[V_{0}\right]_{\mathrm{th}}=\frac{\hbar^{2}}{2 m a^{2}}\left(\frac{\pi}{2}\right)^{2} C, \quad C=1.0 .
$$

We use the eigenvalue integral (2.23) to compute $E_{0}$. It is worthwhile to try several seeds to get some notion of how $E_{0}$ depends on $F$. Here are four choices:

$$
\begin{gathered}
F_{1}(r)=\left\{\begin{array}{cc}
1-\left(K+1 / r_{0}\right) r & \left(r<r_{0}\right) \\
-K r-\ln \left(r / r_{0}\right) & \left(r>r_{0}\right)
\end{array}\right. \\
F_{2}(r)=-K r-\ln \sqrt{\left(r / r_{0}\right)^{2}+1}, \\
F_{3}(r)=-K r-\ln \left(r / r_{0}+1\right), \\
F_{4}(r)=-K r .
\end{gathered}
$$

( $F_{1}$ is chosen so that the function and its derivative are continuous at $r=r_{0}$.) The first three choices depend on a pa- 
TABLE I. The optimum values of $r_{0} / a$, the threshold potential strength, and the coefficient $C$ for the four choices for $F(r)$.

\begin{tabular}{cccc}
\hline \hline$F(r)$ & $r_{0} / a$ & $2 m a^{2}\left[V_{0}\right]_{\mathrm{th}} / \hbar^{2}$ & $C$ \\
\hline$S(r)$ (exact) & & 2.47 & 1.0 \\
$F_{1}(r)$ & 1.38 & 2.54 & 1.03 \\
$F_{2}(r)$ & 0.923 & 2.69 & 1.09 \\
$F_{3}(r)$ & 0.638 & 2.81 & 1.14 \\
$F_{4}(r)$ & & 2.91 & 1.19 \\
\hline \hline
\end{tabular}

rameter $r_{0}$ which determines where the logarithmic term switches on. The fourth choice is particularly crude and omits the logarithm and therefore does not have the known asymptotic behavior of Eq. (1.3).

A computationally simple way of comparing seeds is to obtain the threshold potential strength for the different cases. The results are summarized in Table I.

Note that $E_{0}=\mathcal{E}_{0}$ is a variational energy; in each case parameters $K a$ and $r_{0} / a$ have been chosen to minimize the threshold potential strength, which is equivalent to minimizing $E_{0}$. We see that the results are sensitive to $F . F_{1}$ is the best of our choices; it gives a threshold potential strength that is only $3 \%$ high, so we examine $F_{1}$ in more detail.

To make the exploration, we choose a potential strength well above threshold: $2 m a^{2} V_{0} / \hbar^{2}=3.0$. The bound-state energy is given by Eq. (3.2) to be $E=-0.06132 \hbar^{2} / 2 m a^{2}$. We find that at this potential strength $E_{0}$ is minimized for $r_{0} / a=1.505$ (in the threshold calculation the value was 1.38). We find $E_{0}=-0.0379 \hbar^{2} / 2 m a^{2}$. A measure of the (mediocre) quality of this result is the ratio $E_{0} / E=0.619$.

We next compute $E_{1}$. For this one degree-of-freedom problem, we use Eq. (2.2) because a simple formula for $d T_{n} / d r$ is available:

$$
\begin{aligned}
\frac{d T_{n}}{d r}= & \frac{e^{-2 F(r)}}{r^{2}} \int_{0}^{r} d x x^{2} e^{2 F(x)}\left\{\frac{2 m}{\hbar^{2}}\left[V(x)-E_{n}\right]-F^{\prime \prime}(x)\right. \\
& \left.-\frac{2}{x} F^{\prime}(x)-\left[F^{\prime}(x)\right]^{2}-\left[T_{n-1}^{\prime}(x)\right]^{2}\right\} .
\end{aligned}
$$

We now obtain much improved results: $E_{1}$ $=-0.0589 \hbar^{2} / 2 m a^{2}$, and $E_{1} / E=0.960$. Almost $90 \%$ of the error in $E_{0}$ has been removed in $E_{1}$. These results are tabulated in Table II.

The threshold energies and $E_{0}$ are sensitive to the choice of the seed. However, it is not obligatory to search for a high-quality seed. It may be preferable to use a very simple seed if that allows higher modified WKB approximants to be

TABLE II. Modified WKB corrections for $F_{1}(r)$ and $F_{4}(r)$.

\begin{tabular}{ccc}
\hline \hline$F(r)$ & Energy & Energy/E(exact) \\
\hline$F_{1}(r)$ & $E_{0}=-0.0379 \hbar^{2} / 2 m a^{2}$ & 0.619 \\
& $E_{1}=-0.0589 \hbar^{2} / 2 m a^{2}$ & 0.960 \\
$F_{4}(r)$ & $E_{0}=-0.0109 \hbar^{2} / 2 m a^{2}$ & 0.179 \\
& $E_{1}=-0.0549 \hbar^{2} / 2 m a^{2}$ & 0.895 \\
& $E_{2}=-0.0598 \hbar^{2} / 2 m a^{2}$ & 0.975 \\
& $\mathcal{E}_{2}=-0.06113 \hbar^{2} / 2 m a^{2}$ & 0.9969 \\
\hline \hline
\end{tabular}

computed. In our case, the worst seed, $F_{4}$, has the virtue that $d T_{0} / d r$ involves nothing more exotic than exponential functions, and it is easy to compute higher approximations. At the same time, $F_{4}$ is manifestly inaccurate. It has incorrect asymptotic behavior, and because of this, at our selected potential strength $2 m a^{2} V_{0} / \hbar^{2}=3.0$ we are barely above threshold for this seed. The initial energy reflects this: $E_{0}=0.0109 \hbar^{2} / 2 m a^{2}$, and the ratio $E_{0} / E=0.179$ is terrible. When we compute $E_{1}$ we find $E_{1}=0.0549 \hbar^{2} / 2 m a^{2}$, and $E_{1} / E=0.895$. The second modified WKB approximant for $F_{4}$ shows further improvement: $E_{2}=0.0598 \hbar^{2} / 2 m a^{2}$; $E_{2} / E=0.975$.

As we mentioned in Sec. II, at any stage we have the option to use the action correction $T_{n-1}$ to calculate the variational energy $\mathcal{E}_{n}$ as well as the modified WKB energy $E_{n}$. When we do this for $F_{4}$ we obtain results that confirm the higher accuracy of the variational energy: $\mathcal{E}_{2}=0.06113 \hbar^{2} / 2 m a^{2} ; \mathcal{E}_{2} / E=0.9969$. In this case, at least, good results can be obtained with a poor seed. The results for $F_{4}$ are recorded in Table II.

One of the properties of the modified WKB approximation we have emphasized is that it can be applied to potential problems where $V(\mathbf{r})$ has a general dependence on $\mathbf{r}$. This statement is qualified by the requirement that $F(\mathbf{r})$ be such that Eq. (2.9) can be solved for $G$. One case where this can be done is when $V$ is nearly spherically symmetric. Then we choose $F$ to depend on $r$, relying on the $T_{n}$ to supply the nonspherical corrections. Note that the modified WKB method is more flexible than the method of separation of coordinates in that it is not necessary for surfaces of constant potential to exactly fall on surfaces of constant coordinate. Approximate coincidence suffices. Below we use separation of coordinates to construct $G$ in spherical coordinates.

When $F$ depends only on $r$, we write $G$ in the form

$$
G\left(\mathbf{r}, \mathbf{r}^{\prime}\right)=\sum_{\ell=0}^{\infty} g_{\ell}\left(r, r^{\prime}\right) \sum_{m=-\ell}^{m=\ell} Y_{\ell m}(\theta, \phi) Y_{\ell m}^{*}\left(\theta^{\prime}, \phi^{\prime}\right),
$$

where the radial Green function satisfies

$$
\begin{aligned}
& \frac{\partial^{2} g \ell\left(r, r^{\prime}\right)}{\partial r^{2}}+2\left(\frac{1}{r}+\frac{d F}{d r}\right) \frac{\partial g \ell\left(r, r^{\prime}\right)}{\partial r}-\frac{\ell(\ell+1)}{r^{2}} g\left(r, r^{\prime}\right) \\
& =\frac{1}{r^{2}} \delta\left(r-r^{\prime}\right)
\end{aligned}
$$

This Green function is

$$
g_{\ell}\left(r, r^{\prime}\right)=R_{\ell}^{+}\left(r_{>}\right) R_{\ell}^{-}\left(r_{<}\right) e^{2 F\left(r^{\prime}\right)} / C_{\ell},
$$

where $R^{+,-}$satisfy the homogeneous equation

$$
\frac{d^{2} R \ell}{d r^{2}}+2\left(\frac{1}{r}+\frac{d F}{d r}\right) \frac{d R_{\ell}}{d r}-\frac{\ell(\ell+1)}{r^{2}} R_{\ell}=0 .
$$

The factor $C_{\ell}$ comes from the Wronskian $W\left(R_{\ell}^{+}, R_{\ell}^{-}\right)=C_{\ell} e^{-2 F(r) / r^{2}}$.

For $\ell=0$, Eq. (3.9) has the same solutions we found for the case of spherical symmetry, and we can choose $R_{0}^{+,-}=R^{+,-}$of Eq. (2.19). We again encounter the eigen- 
value integral (2.23) with this change: Only the $s$-wave projection of $V$ enters into the determination of $E_{0}$. This is in keeping with our assumption that $V$ is nearly spherically symmetric. The higher $E_{n}$ 's receive contributions from all of the angular projections of $V$.

The solutions of Eq. (3.9) for $\ell>0$ require further analysis. We first note that near $r=0$ they behave like $r^{\prime}$ or $r^{-\ell-1}$, and we must choose $R_{\ell}^{-}$to have the nonsingular behavior $r^{\prime}$. At large $r$, solutions of Eq. (3.9) behave like those of Eq. (2.19) because the last term in Eq. (3.9) is unimportant at large $r$. We must choose the solution behaving like a constant so that $R^{+}$remains finite at large $r$.

In the case of $F_{1}(r)$, Eq. (3.9) has solutions that are familiar functions. In the interval $r<r_{0}$, these are confluent hypergeometic functions:

$$
\begin{gathered}
R_{\ell 1}=r^{\ell} \Phi\left(\ell, 2 \ell+2,2 K r+2 r / r_{0}\right), \\
R_{\ell 2}=r^{-\ell-1} \Phi\left(-\ell-1,-2 \ell, 2 K r+2 r / r_{0}\right) .
\end{gathered}
$$

In the interval $r>r_{0}$ they are modified spherical Bessel functions:

$$
R_{/ 3}=\sqrt{r} e^{K r} I_{\ell+1 / 2}(K r), \quad R_{\ell 4}=\sqrt{r} e^{K r} K_{\ell+1 / 2}(K r) .
$$

We then have the solutions

$$
\begin{aligned}
& R_{\ell}^{-}=\left\{\begin{array}{ll}
R_{\ell 1}, & r<r_{0} \\
\alpha R_{\ell 3}+\beta R_{\ell 4}, & r>r_{0}
\end{array},\right. \\
& R_{\ell}^{+}= \begin{cases}\gamma R_{\ell 1}+\delta R_{\ell 2}, & r<r_{0} \\
R_{\ell 4}, & r>r_{0}\end{cases}
\end{aligned}
$$

The coefficients are fixed by demanding that the functions and their first derivatives be continuous at $r=r_{0}$; they may be expressed in terms of Wronskians evaluated at $r=r_{0}$.

When a particle moves under the influence of a highly nonspherical potential, the Green function should be constructed in coordinates chosen so it is reasonable for $F$ to depend only on one of them, $\xi$. As an example, consider a potential well in the shape of a right circular cylinder of diameter $D$ and length $L$ :

$$
V(\mathbf{r})=\left\{\begin{array}{l}
-V_{0}, \quad|z|<L / 2, \quad \sqrt{x^{2}+y^{2}}<D / 2 \\
0 \quad \text { otherwise. }
\end{array}\right.
$$

If $L \sim D$, this is an example of an approximately spherical potential, and it is appropriate to use the Green function constructed above. Here we consider the case of a long thin rod: $L \gg D$. Now it is manifestly inadequate to take the surfaces of constant $F$ to be spherical. These surfaces ought to be figures of rotation about the $z$ axis in the form of elongated cigars: prolate spheroids. This suggests use of the prolate spheroidal coordinate system:

$$
\begin{gathered}
x=\frac{r_{0}}{2} \sqrt{\xi^{2}-1} \sin \theta \cos \phi, \\
y=\frac{r_{0}}{2} \sqrt{\xi^{2}-1} \sin \theta \sin \phi, \\
z=\frac{r_{0}}{2} \xi \cos \theta, \\
1 \leqslant \xi<\infty, \quad 0 \leqslant \theta \leqslant \pi, \quad 0 \leqslant \phi<2 \pi, \\
d V=\left(\frac{r_{0}}{2}\right)^{3}\left(\xi^{2}-\cos ^{2} \theta\right) \sin \theta d \xi d \theta d \phi .
\end{gathered}
$$

Surfaces of constant $\xi$ are ellipsoids of revolution about the $z$ axis, the ellipses having foci at $z= \pm r_{0} / 2$. If $r_{0}$ is appropriately chosen (by a variational minimization of $E_{0}$ ), the shape of the ellipsoids can be made to resemble that of the rod for $\xi \sim 1$; for large $\xi$ the ellipsoids approach spheres of radius $r_{0} \xi / 2$. An obvious choice for $F$ is

$$
F(\xi)=-K r_{0} \xi / 2-\ln \xi .
$$

In prolate spheroidal coordinates, Eq. (2.9) takes the form

$$
\begin{gathered}
\frac{\partial}{\partial \xi}\left[\left(\xi^{2}-1\right) \frac{\partial G}{\partial \xi}\right]+\frac{1}{\sin \theta} \frac{\partial}{\partial \theta}\left[\sin \theta \frac{\partial G}{\partial \theta}\right]+\left[\frac{1}{\xi^{2}-1}\right. \\
\left.+\frac{1}{\sin ^{2} \theta}\right] \frac{\partial^{2} G}{\partial \phi^{2}}+2\left(\xi^{2}-1\right) \frac{d F}{d \xi} \frac{\partial G}{\partial \xi} \\
=\frac{2}{r_{0}} \delta\left(\xi-\xi^{\prime}\right) \delta\left(\cos \theta-\cos \theta^{\prime}\right) \delta\left(\phi-\phi^{\prime}\right) .
\end{gathered}
$$

The solution has the form

$$
\begin{aligned}
& G\left(\xi, \theta, \phi ; \xi^{\prime}, \theta^{\prime}, \phi^{\prime}\right) \\
& \quad=\sum_{\ell=0}^{\infty} \sum_{m=-\ell}^{\ell} g_{\ell, m}\left(\xi, \xi^{\prime}\right) Y_{\ell m}(\theta, \phi) Y_{\ell m}^{*}\left(\theta^{\prime} \phi^{\prime}\right),
\end{aligned}
$$

where the radial Green function satisfies

$$
\begin{gathered}
\left(\xi^{2}-1\right) \frac{\partial^{2} g_{\ell m}}{\partial \xi^{2}}+2\left[\xi+\left(\xi^{2}-1\right) \frac{d F}{d \xi}\right] \frac{\partial g_{\ell m}}{\partial \xi} \\
-\left[\ell(\ell+1)+\frac{m^{2}}{\xi^{2}-1}\right] g_{\ell m}=\frac{2}{r_{0}} \delta\left(\xi-\xi^{\prime}\right) .
\end{gathered}
$$

The radial Green function is

$g_{\ell m}\left(\xi, \xi^{\prime}\right)=\frac{2}{r_{0} C_{\ell m}} e^{2 F\left(\xi^{\prime}\right)} \Xi_{\ell m}^{-}\left(\xi_{<}\right) \Xi_{\ell m}^{+}\left(\xi_{>}\right)$.

$C_{\ell m}$ appears in the Wronskian $W\left(\Xi^{+}, \Xi^{-}\right)$ $=C_{\ell m} e^{-2 F} /\left(\xi^{2}-1\right)$. The functions $\Xi_{\ell m}^{ \pm}$satisfy the homogeneous equation obtained by removing the $\delta$ function source in Eq. (3.18). 
The general method of constructing the Green function appropriate to the rod-shaped potential well is now clear. We discontinue discussion of the functions $\Xi \pm / m$ except to point out that the differential equation they satisfy has three regular singular points and an irregular singular point at infinity.

\section{LONG-RANGE POTENTIALS}

When a potential is long range and behaves at large distance like $r^{-p}, 1<p<2$, there is no longer a threshold strength for the bound state [7]. The modified WKB approximation reproduces this result, and it does so in lowest order, when $n=0$. We demonstrate this by showing that with the choices of $F$ given by Eq. (3.4) we can adjust $r_{0}$ so that $E_{0}=0$ no matter how weak the strength of the potential tail. A futher adjustment of $r_{0}$ will then produce a negative (bound-state) energy.

We consider the case of $F_{2}$. For the potential $V_{0} / r^{p}$ $(r>a)$, the eigenvalue equation implied by Eq. (2.23) takes the form, when $n=E_{0}=0$ :

$$
0=-\frac{2 m V_{0}}{\hbar^{2}} \int_{a}^{\infty} \frac{r^{2-p} d r}{r^{2}+r_{0}^{2}}+3 r_{0}^{2} \int_{0}^{\infty} \frac{r^{2} d r}{\left(r^{2}+r_{0}\right)^{3}}
$$

This may be written

$$
\begin{aligned}
0= & -\frac{m V_{0} r_{0}^{1-p}}{\hbar^{2}} \frac{2 \pi}{2 \sin \pi(p-1) / 2} \\
& -\sum_{k=0}^{\infty}\left(\frac{a}{r_{0}}\right)^{2 k+3-p} \frac{(-1)^{k}}{k+(3-p) / 2}+\frac{3 \pi}{16 r_{0}} .
\end{aligned}
$$

For $1<p<2$, there is some large value of $r_{0}$ for which this equation is satisfied, no matter how small $V_{0}$ may be. There must be a nodeless bound state.

When $p=1$, we come to the classic case of the Coulomb potential. At this point, the asymptotic behavior of the wave function changes, and in three dimensions the coefficient of the $\ln \left(r / r_{0}\right)$ term depends on the potential strength and bound-state energy. Since this coefficient is no longer known, the obvious response is to treat this coefficient as an additional parameter, $r_{1}$. Thus, for example, $F_{1}$ is generalized to

$$
F_{1}(r)=\left\{\begin{array}{l}
r_{1}-\left(K+\frac{r_{1}}{r_{0}}\right) r \quad\left(r<r_{0}\right) \\
-K r-r_{1} \ln \left(r / r_{0}\right) \quad\left(r>r_{0}\right) .
\end{array}\right.
$$

But now the determination of the variational parameters is obvious, because with $r_{1}=0, F_{1}=-K r=S$. Therefore $T(r)=0$, and we immediately have the hydrogen wave function, with $E_{0}=E$. The same result is achieved with $F_{2}$, $F_{3}$, and $F_{4}$.

It is fortuitous that the hydrogen wave function occurs among the natural choices for $F$. Still, it is nice that the Coulomb potential is so easily encompassed by the modified WKB approximation.

\section{V. $\phi^{4}$ FIELD THEORY}

The modified WKB approximation can be applied to problems having many degrees of freedom. We illustrate this using the case of $\phi^{4}$ field theory in one spatial dimension. In the continuum the Hamiltonian is

$$
\begin{aligned}
H= & \int_{0}^{L} d x\left\{\frac{1}{2} c^{2} \pi^{2}(x)+\frac{1}{2}\left[\frac{d \phi(x)}{d x}\right]^{2}\right. \\
& \left.+\frac{1}{2} \frac{c^{2}}{\hbar^{2}}\left(m^{2}+\delta m^{2}\right) \phi^{2}(x)+\frac{\lambda}{24} \phi^{4}(x)\right\} .
\end{aligned}
$$

We identify fields at $x=0$ and $L$. To treat the system by the modified WKB method we must deal with a discrete set of degrees of freedom. We therefore divide the line into $N$ segments of length $a ; N=L / a$. Each segment is represented by a dimensionless lattice coordinate $\phi_{k}, k=1, \ldots, N$. The lattice Hamiltonian is

$$
\begin{aligned}
H= & \frac{\hbar c}{a} \sum_{k=1}^{N}\left[-\frac{1}{2} \frac{\partial^{2}}{\partial \phi_{k}^{2}}+\frac{1}{2}\left(\phi_{k+1}-\phi_{k}\right)^{2}\right. \\
& \left.+\frac{1}{2}\left(\frac{c a}{\hbar}\right)^{2}\left(m^{2}+\delta m^{2}\right) \phi_{k}^{2} \frac{\lambda \hbar c a}{24} \phi_{k}^{4}\right]
\end{aligned}
$$

Putting the field theory on the lattice introduces a shortdistance cutoff $a$, or equivalently a large momentum cutoff $\hbar / a$. This removes the notorious divergences of continuum quantum field theory. However, the divergences still lurk and reveal themselves when we approach the continuum limit by taking $a \rightarrow 0, N \rightarrow \infty$, with $N a=L$ fixed. We want to be able to take the limit, of course, since for us the lattice is only a computational device. In our case we find that as we take $a \rightarrow 0$ we approach a continuum theory having infinite mass. (We show this below.) This pathology disappears when we include an appropriate mass counterterm in the Hamiltonian:

$$
\begin{gathered}
\delta m^{2}=-\frac{\lambda \hbar}{4 c} \Delta_{0}, \quad \Delta_{0}=\frac{1}{N} \sum_{p=1}^{N} \frac{1}{\omega_{0}(p)}, \\
\omega_{0}(p)=\left[\left(\frac{m c a}{\hbar}\right)^{2}+4 \sin ^{2}\left(\frac{\pi p}{N}\right)\right]^{1 / 2} .
\end{gathered}
$$

This counterterm is the lattice version of the very counterterm that must be included in the continuum Hamiltonian. At small $a, \Delta_{0}$ grows logarithmically to keep the effective mass finite.

$$
\Delta_{0} \sim \frac{1}{\pi} \ln \frac{\hbar^{2}}{m^{2} c^{2} a^{2}}
$$

In one spatial dimension $\phi^{4}$ field theory is superrenormalizable [8]. For this system, the explicit counterterm in (5.3) suffices to remove all $a \rightarrow 0$ divergences in "physical', entities like the correlator

$$
\langle 0|\phi(0) \phi(x)| 0\rangle \sim \hbar c\left\langle 0\left|\frac{1}{N} \sum_{k=1}^{N} \phi_{k} \phi_{k+x / a}\right| 0\right\rangle .
$$


Unfortunately, the ground-state energy $E$ that has figured prominently in the modified WKB approximation is not "physical" in the sense used here. Even free field theory, $\lambda=0$, includes zero-point energies for each degree of freedom, and when summed these diverge as $a \rightarrow 0$. We will cope with $E$ as we go along. This point aside, Eqs. (5.2), (5.3) define a lattice representation of continuum $\phi^{4}$ field theory to which we can apply the modified WKB approximation.

A central issue for this many-degree-of-freedom problem is finding an $F$ that is appropriate, and for which the multidimensional integrals can be evaluated and the Green function found. There is just one choice for which this is easy, the Gaussian

$$
F=-\frac{1}{2} \sum_{k_{1}, k_{2}} M_{k_{1} k_{2}} \phi_{k_{1}} \phi_{k_{2}} \equiv-\frac{1}{2} \phi M \phi
$$

with $M$ a real symmetric matrix. Consider the eigenvalue equation for $E_{0}$.

$$
\begin{aligned}
0= & \int(\Pi d \phi) e^{-\phi M \phi}\left\{\sum _ { k } \left[\left(\phi_{k+1}-\phi_{k}\right)^{2}\right.\right. \\
& \left.+(c a / \hbar)^{2}\left(m^{2}+\delta m^{2}\right) \phi_{k}^{2}+\left(\lambda \hbar c a^{2}\right) \phi_{k}^{4} / 12\right] \\
& \left.-2 E_{0} a / \hbar c+\operatorname{Tr}(M)-\phi M^{2} \phi\right\}
\end{aligned}
$$

Every term in the large curly brackets involves powers of $\phi_{k}$, so the integral can be evaluated by taking derivatives with respect to $\alpha_{k}$ of the generating function

$$
I_{1}(\alpha)=\int(\Pi d \phi) \exp (-\phi M \phi+i \alpha \phi), \quad \alpha \phi \equiv \sum_{k} \alpha_{k} \phi_{k} .
$$

This generator, in turn, can be evaluated easily by a change of coordinates. Let the eigenvectors and eigenvalues of $M$ be $v^{q}$ and $\mu^{q}$.

$$
M v^{q}=\mu^{q} v^{q} \quad(q=1, \ldots, N) .
$$

The new coordinates are

$$
\eta^{q}=\sum_{k} \phi_{k} v_{k}^{q}, \quad \phi_{k}=\sum_{q} \eta^{q} v_{k}^{q}
$$

In these coordinates $I_{1}$ becomes a product of $N$ independent Gaussian integrals, and

$$
I_{1}(\alpha)=\frac{\pi^{N / 2}}{\sqrt{\operatorname{det} M}} \exp \left(-\frac{1}{4} \alpha M^{-1} \alpha\right) .
$$

Equation (5.7) becomes

$$
\begin{aligned}
0= & I_{1}(0)\left\{\sum _ { k } \left[M_{k k}^{-1}-M_{k, k+1}^{-1}+(c a / \hbar)^{2}\left(m^{2}+\delta m^{2}\right) M_{k k}^{-1} / 2\right.\right. \\
& \left.+\left(\lambda \hbar c a^{2}\right)\left(M_{k k}^{-1}\right)^{2} / 16\right]-2 E_{0} a / \hbar c+\operatorname{Tr}(M) \\
& \left.-\operatorname{Tr}\left(M^{2} M^{-1}\right)\right\} .
\end{aligned}
$$

$M_{k_{1} k_{2}}$ must be a function of $k_{1}-k_{2}$ of period $N$ owing to the homogeneity and periodicity of the Hamiltonian. It can be written as a discrete Fourier transform.

$$
M_{k_{1} k_{2}}^{ \pm 1}=\frac{1}{N} \sum_{p=1}^{N} \omega^{ \pm 1}(p) \exp \left[\frac{2 \pi i p\left(k_{1}-k_{2}\right)}{N}\right] .
$$

With this the eigenvalue equation decouples into modes.

$$
\begin{aligned}
0= & I_{1}(0)\left\{\sum _ { p } \left[\frac{\omega(p)}{2}+\frac{1}{\omega(p)}\left(1-\cos \frac{2 \pi p}{N}\right.\right.\right. \\
& \left.\left.+(c a / \hbar)^{2}\left(m^{2}+\delta m^{2}\right)\right)\right] \\
& \left.+N\left(\lambda \hbar c a^{2}\right)\left[\frac{1}{N} \sum_{p} \frac{1}{\omega(p)}\right]^{2}-\frac{2 E_{0} a}{\hbar c}\right\} .
\end{aligned}
$$

The $\omega(p)$ are parameters in $M$ that are fixed by minimizing $E_{0}$. We find

$$
\begin{gathered}
\omega(p)=\left[\left(\frac{c a}{\hbar}\right)^{2}\left(m^{2}+\delta m^{2}\right)+\frac{\lambda \hbar c a^{2}}{4} \Delta+\sin ^{2}\left(\frac{\pi p}{N}\right)\right]^{1 / 2} \\
\Delta=\frac{1}{N} \sum_{p} \frac{1}{\omega(p)} .
\end{gathered}
$$

Equation (5.16) determines $\Delta$, and it is enlightening to examine its solution near the continuum limit when we drop the counterterm $\delta m^{2}$. Following Eq. (5.4),

$$
\Delta \sim \frac{1}{\pi} \ln \frac{4}{\lambda \hbar c a^{2} \Delta}, \quad \Delta \sim \frac{1}{\pi} \ln \frac{4}{\lambda \hbar c a^{2}}+O\left(\ln \ln \frac{4}{\lambda \hbar c a^{2}}\right) .
$$

Therefore the square of the effective mass in $\omega(p)$ becomes, in the limit of small $a$,

$$
m^{2}+\frac{\lambda \hbar^{3} \Delta}{4 c} \sim m^{2}+\frac{\lambda \hbar^{3}}{4 \pi c} \ln \frac{4}{\lambda \hbar c a^{2}} .
$$

This shows that we approach a continuum theory having infinite mass. On the other hand, when we do include the counterterm, Eq. (5.16) has the solution $\Delta=\Delta_{0}$; $\omega(p)=\omega_{0}(p)$. Then the mode function $\omega(p)$ never changes, no matter what the lattice spacing or coupling strength.

The energy $E_{0}$ is

$$
\frac{E_{0}}{L}=\frac{\hbar c}{2 a^{2}}\left[\frac{1}{N} \sum_{p=1}^{N} \omega(p)\right]-\frac{m^{2} c^{3}}{\hbar}\left(\frac{\lambda \hbar^{3}}{m^{2} c}\right) \Delta_{0}^{2} .
$$

The sum on the right is the zero-point energy of the degrees of freedom. It is present in free field theory, and its contribution is quadratically divergent in $a^{-1}$. The last term, proportional to the dimensionless coupling $\lambda \hbar^{3} / m^{2} c$, diverges like $\left(\ln a^{-1}\right)^{2}$. In perturbation theory it arises because anomalous combinatorics spoil the cancellation of divergences in self-energy loops and $\Delta_{0}$. This failure to cancel occurs only at order $\lambda$ of perturbation theory, so $E_{0}$ already exhibits all the terms that diverge as $a \rightarrow 0$. 
To compute $E_{1}$ we need the Green function satisfying

$\left(\frac{\partial^{2}}{\partial \phi^{2}}-2 \phi M \frac{\partial}{\partial \phi}\right) G\left(\phi, \phi^{\prime}\right)=\prod_{k} \delta\left(\phi_{k}-\phi_{k}^{\prime}\right)$,

in matrix notation. We use Eq. (2.12) to construct $G$, which requires us to construct eigenfunctions of the operator on the left. We again use coordinates $\eta$; then the equation for the eigenfunctions and eigenvalue is

$$
\sum_{q=1}^{N}\left[\frac{\partial^{2}}{\partial\left(\eta^{q}\right)^{2}}-2 \mu^{q} \eta^{q} \frac{\partial}{\partial \eta^{q}}\right] \psi=\mu \psi
$$

This equation may be solved by separation of variables.

$$
\psi_{\{s\}}=\prod_{q=1}^{N} H_{s_{q}}\left(\eta^{q} \sqrt{\mu^{q}}\right) .
$$

The functions $H_{s}$ are Hermite polynomials satisfying

$$
\begin{gathered}
\frac{d^{2} H_{s}(x)}{d x^{2}}-2 x \frac{d H_{s}(x)}{d x}+2 s H_{s}(x)=0, \\
\int_{-\infty}^{\infty} d x e^{-x^{2} H_{s_{1}}(x) H_{s_{2}}(x)=\delta_{s_{1}, s_{2}} \sqrt{\pi} 2^{s_{1}}\left(s_{1} !\right) .}
\end{gathered}
$$

There are $N$ indices on our wave function, and the eigenvalue is a linear function of them:

$$
\mu=-2 \sum_{q=1}^{N} s_{q} \mu^{q}
$$

The Green function is

$$
\begin{aligned}
G= & \left(-\frac{1}{2}\right) e^{-\phi^{\prime} M \phi^{\prime}} \int_{0}^{1} \frac{d z}{z} \prod_{q=1}^{N}\left(\frac{\mu_{q}}{\pi}\right)^{1 / 2} \\
& \times\left[\sum_{s_{q}=0}^{\infty}\left(\frac{z^{\mu_{q}}}{2}\right)^{s_{q}} \frac{H_{s_{q}}\left(\eta^{q} \sqrt{\mu^{q}}\right) H_{s_{q}}\left(\eta^{q \prime} \sqrt{\mu^{q}}\right)}{\left(s_{q} !\right)}\right] .
\end{aligned}
$$

The purpose of the integration over $z$ is to produce the denominator $1 / \mu_{k}$ in Eq. (2.12). The sum over $s_{q}$ is given by Mehler's formula [9]. Reverting to the original variables,

$$
\begin{aligned}
G\left(\phi, \phi^{\prime}\right)= & -\frac{1 \sqrt{\operatorname{det}(M)}}{2 \pi^{N / 2}} \int_{\epsilon}^{1} \frac{d z}{z \sqrt{\operatorname{det}\left(1-z^{2 M}\right)}} \\
& \times \exp \left[-\phi^{\prime} \frac{M}{1-z^{2 M}} \phi^{\prime}-\phi \frac{M z^{2 M}}{1-z^{2 M}} \phi\right. \\
& \left.+2 \phi^{\prime} \frac{M z^{M}}{1-z^{2 M}} \phi\right] .
\end{aligned}
$$

As we expect from the discussion of Sec. II, this Green function does not exist because $\mu=0$ is an eigenvalue. In Eq. (5.26) the divergence appears at $z=0$, and it has been regulated by a cutoff at $z=\epsilon$. However, when computing $T_{n}$, the coefficient of the $1 / z$ factor is

$$
\begin{aligned}
& \int\left(\prod d \phi\right) e^{-\phi M \phi} D_{n}(\phi) \\
& D_{n}(\phi)=\left\{\sum _ { k } \left[\left(\phi_{k+1}-\phi_{k}\right)^{2}+\left(\frac{c a}{\hbar}\right)^{2}\left(m^{2}+\delta m^{2}\right) \phi_{k}^{2}\right.\right. \\
&\left.+\frac{\lambda \hbar c a^{2}}{12} \phi_{k}^{4}\right]-\frac{2 a E_{n}}{\hbar c} \\
&\left.+\operatorname{Tr}(M)-\phi M^{2} \phi-\left(\frac{\partial T_{n-1}}{\partial \phi}\right)^{2}\right\}
\end{aligned}
$$

Setting this to zero, we obtain a finite $T_{n}$ as $\epsilon \longrightarrow 0$. Thus we again encounter the eigenvalue equation as a consistency condition for the solution of Schrödinger's equation.

The dependencies on the fields in Eq. (5.26) are Gaussian, so we can use $I_{1}$ to evaluate the generating function

$$
\begin{aligned}
I_{2}(\alpha, \beta)= & \int\left(\prod d \phi d \phi^{\prime}\right) G\left(\phi, \phi^{\prime}\right) \\
& \times \exp \left(-\phi M \phi+i \alpha \phi+i \beta \phi^{\prime}\right) \\
= & \frac{\pi^{N / 2}}{\sqrt{\operatorname{det}(M)}} \exp \left(-\frac{1}{4} \alpha M^{-1} \alpha-\frac{1}{4} \beta M^{-1} \beta\right) \\
& \times\left(-\frac{1}{2}\right) \int_{\epsilon}^{1} \frac{d z}{z} \exp \left(-\frac{1}{2} \alpha\left[z^{M} M^{-1}\right] \beta\right) .
\end{aligned}
$$

By differentiation of $I_{2}$ we obtain all the contributions in

$$
E_{1}-E_{0}=\frac{\hbar c}{2 a} \frac{\int\left(\Pi d \phi d \phi^{\prime}\right) e^{-\phi M \phi} G\left(\phi, \phi^{\prime}\right) D(\phi) D\left(\phi^{\prime}\right)}{\int(\Pi d \phi) e^{-\phi M \phi}} .
$$

The result is

$$
\frac{E_{1}-E_{0}}{L}=-\frac{m^{2} c^{3}}{\hbar}\left(\frac{\lambda \hbar^{3}}{m^{2} c}\right)^{2} \frac{\Delta_{2}}{384}
$$

where

$$
\Delta_{2}=\left(\frac{m c a}{\hbar}\right)^{2} \int_{0}^{1} \frac{d z}{z} \frac{1}{N}\left[\sum_{k_{1}, k_{2}}\left(z^{M} M^{-1}\right)_{k_{1}, k_{2}}\right]^{4}
$$

Using Eq. (5.13),

$\left(z^{M} M^{-1}\right)_{k_{1}, k_{2}}=\frac{1}{N} \sum_{p=1}^{N} \frac{z^{\omega_{0}(p)}}{\omega_{0}(p)} \exp \left[\frac{2 \pi i p\left(k_{1}-k_{2}\right)}{N}\right]$, 


$$
\begin{aligned}
\Delta_{2}= & \left(\frac{m c a}{\hbar}\right)^{2}\left(\frac{1}{N}\right)^{3} \\
& \times \sum_{p_{1}, p_{2}, p_{3}} \frac{1}{\omega_{0}\left(p_{1}\right) \omega_{0}\left(p_{2}\right) \omega_{0}\left(p_{3}\right) \omega_{0}\left(p_{1}+p_{2}+p_{3}\right)} \\
& \times \frac{1}{\left[\omega_{0}\left(p_{1}\right)+\omega_{0}\left(p_{2}\right)+\omega_{0}\left(p_{3}\right)+\omega_{0}\left(p_{1}+p_{2}+p_{3}\right)\right]} .
\end{aligned}
$$

It is straightforward to verify that $\Delta_{2}$ remains finite as $a \rightarrow 0$.

The expression for $\Delta_{2}$ is the three-loop vacuum bubble graph of second-order perturbation theory (in "oldfashioned" form). Thus it seems that what emerges is simply perturbation theory. Such a result is plausible because our $F$ is the $S$ of free field theory, and we compute corrections to that. However, the result for $E_{1}-E_{0}$ is deceptive; what we obtain is not straightforward perturbation theory, but a reordering of perturbation theory.

Convenient equations for counting powers of $\lambda$ are

$$
\begin{gathered}
E_{n}-E_{n-1}=\frac{\int(\Pi d \phi) e^{2 F}\left\{\left[\partial\left(T_{n-2}-T_{n-1}\right) / \partial \phi\right]\left[\partial\left(T_{n-2}+T_{n-1}\right) / \partial \phi\right]\right\}}{\int(\Pi d \phi) e^{2 F}}, \\
T_{n}(\phi)-T_{n-1}(\phi)=\int\left(\Pi d \phi^{\prime}\right) G\left(\phi, \phi^{\prime}\right)\left((2 a / \hbar c)\left(E_{n-1}-E_{n}\right)+\left\{\left[\partial\left(T_{n-2}-T_{n-1}\right) / \partial \phi^{\prime}\right]\left[\partial\left(T_{n-2}+T_{n-1}\right) / \partial \phi^{\prime}\right]\right\}\right) .
\end{gathered}
$$

We have established directly that $E_{1}-E_{0}=O\left(\lambda^{2}\right)$; it follows from the top equation that $T_{0}=O(\lambda)$. The second equation then establishes $T_{1}-T_{0}=O\left(\lambda^{2}\right)$.

The next round of estimates determines $E_{2}-E_{1}$ $=O\left(\lambda^{3}+\lambda^{4}\right)$ because $T_{0}+T_{1}=O\left(\lambda+\lambda^{2}\right)$. By induction one establishes that $T_{n}$ and $E_{n}$ include all perturbative terms up to $O\left(\lambda^{n+1}\right)$ and some of the perturbative terms up to $O\left(\lambda^{p}\right), p=2^{n}$.

The reordering of perturbation theory has its origin in the quadratic form of the gradient terms that the modified WKB approximation treats by successive approximations. We saw in Sec. II that the same nonlinearity is what leads to formal convergence of the approximation. Therefore the reordering of perturbation theory is the means by which the modified WKB approximation "improves" perturbation theory when $\lambda$ is not small.

We learn from our exercise with lattice field theory that calculations can be carried out, and they lead to sensible results. But it is possible, within the modified WKB approximation, to contemplate alternatives that transcend perturbation theory by choosing $F$ to be non-Gaussian. an obvious generalization of $F$ is

$$
\widetilde{F}=\sum_{q=1}^{N}\left[-\frac{1}{2} \widetilde{\mu}^{q}\left(\eta^{q}\right)^{2}-\frac{1}{4} \widetilde{\nu}^{q}\left(\eta^{q}\right)^{4}\right]
$$

We use plane-wave coordinates so that all degrees of freedom are coupled. The mode eigenvalue equation is unfamiliar, and Mehler's formula is not available, so we must work harder. A computer would be required to assemble $G$.

A payoff is possible when a seed like $\widetilde{F}$ is used. Recall that at $\lambda \hbar^{3} / m^{2} c \sim 10, \phi^{4}$ field theory makes a transition to a state of broken $\phi \leftrightarrow-\phi$ symmetry in which the field has a vacuum expectation value: $\langle 0|\phi(x)| 0\rangle=\phi_{0} \neq 0$ [8]. From this fact alone we see that $F$ must be inadequate at strong coupling because with $F, \phi_{0}=0$. (This example shows that although $F$ need not be accurate, as we emphasized in Sec. III, it must belong to the correct class of functions.) But in $\widetilde{F}$, as the variational parameters $\widetilde{\mu}^{q}$ vary smoothly with $\lambda$, a vacuum expectation value can develop beyond a critical coupling.

A trick attributed to Feynman can be applied to this problem to allow the calculation of the vacuum expectation values of operators other than $H$. For example, suppose we wish to compute the correlator of Eq. (5.5). For this purpose augment $H$ by adding a term $\xi(1 / N) \Sigma_{k} \phi_{k} \phi_{k+d}$. The extended Hamiltonian remains homogeneous and periodic, so the techniques we have developed continue to apply. $E$ depends on $\xi$, of course, and by first-order perturbation theory (in $\xi$ ), $d E / d \xi(\xi=0)$ is just the correlator of Eq. (5.5). Of course, we know $E$ only approximately, and we must replace it by $E_{n}$ or $\mathcal{E}_{n}$.

\section{HELIUM}

Because of the Pauli principle, helium is the only multielectron atom for which a symmetric spatial wave function is physical. The nodeless state we construct is the ground state of helium.

The Hamiltonian we study includes only the potential terms representing the electric forces acting on electrons moving around an immobile nucleus. The ground-state energy of the simplified Hamiltonian therefore differs slightly from that of physical helium where magnetic, recoil, and relativistic corrections are present. The Hamiltonian is

$$
H=-\frac{1}{2}\left(\nabla_{1}^{2}+\nabla_{2}^{2}\right)-\frac{2}{r_{1}}-\frac{2}{r_{2}}+\frac{1}{\left|\mathbf{r}_{1}-\mathbf{r}_{2}\right|} .
$$

We use dimensionless coordinates, so the eigenvalues of $H$ must be multiplied by $m e^{4} / \hbar^{2}$. 
A standard textbook variational computation with this Hamiltonian assumes that the wave function is a product of exponential (hydrogenic) factors [10]. The resulting energy, $\langle H\rangle=-(27 / 16)^{2}=-2.848$, is very close to the experimental ground-state energy, -2.90 , and we adopt it as a standard for the problem. Since we have the tools to deal with manybody problems when $F$ is a quadratic form, we use

$$
F=-\frac{1}{2} K_{0}\left(r_{1}^{2}+r_{2}^{2}\right)-K_{1} \mathbf{r}_{1} \cdot \mathbf{r}_{2} .
$$

Our choice of $F$ corresponds to a Gaussian first guess for the helium wave function. We compute $E_{0}$, choosing $K_{0}$ and $K_{1}$ to minimize it and find

$$
E_{0}=-2.324, \quad K_{0}=1.549, \quad K_{1}=(0.09238) K_{0} .
$$

We obtain only 0.816 of the "standard" binding, which means that the modified WKB correction is substantial. An interesting feature of our result is that $K_{1}$ is so small. The repulsion between electrons enhances the probability of finding the electrons on opposite sides of the nucleus, but not by much. In fact, if we set $K_{1}=0$, the binding is decreased by less than $1 \%$, and $E_{0}=-2.301$. We use this version of $F$ when calculating $E_{1}$ because of the resulting simplifications. The computation of $E_{1}$ now parallels that of Sec. V. The main new feature is that the terms in the potential are Coulomb, not polynomial. When we use generating function $I_{2}$, we integrate over parameters to generate the Coulomb terms, using the relation

$$
\frac{1}{r}=\frac{1}{2 \pi^{2}} \int \frac{d^{3} \alpha}{\alpha^{2}} \exp [i \alpha \cdot \mathbf{r}] .
$$

Summarizing our results:

$$
\begin{gathered}
E_{0}=-2.301, \quad E_{1}=-2.707, \quad\langle H\rangle=-2.848, \\
\frac{E_{0}}{\langle H\rangle}=0.808, \quad \frac{E_{1}}{\langle H\rangle}=0.951 .
\end{gathered}
$$

[1] R. A. Leacock and M. J. Padgett, Phys. Rev. Lett. 50, 3 (1983); Phys. Rev. D 28, 2491 (1983).

[2] A. Soares de Castro and A. de Souza Dutra, Found. Phys. 21, 649 (1991).

[3] L. D. Landau and E. M. Lifschitz, Quantum Mechanics, NonRelativistic Theory, 2nd ed. (Pergamon, New York, 1965), pp. 158-187.

[4] J. B. Bronzan, Am. J. Phys. 55, 54 (1987).
Note that the energies $E_{0}$ and $E_{1}$ are closer to $\langle H\rangle$ than were the corresponding energies in the case of the spherical square well. This suggests that convergence for helium is good, and that $\mathcal{E}_{2}$ would differ from the ground-state energy of Hamiltonian (6.1) by a fraction of a percent. Indeed, $\mathcal{E}_{2}$ could lie below $\langle H\rangle$ because the latter is a variational estimate.

\section{CONCLUSIONS}

We have proposed an approximation for nodeless wave functions in quantum mechanics and field theory that assumes the relative importance of gradient and Laplacian terms is the reverse of that in the WKB approximation. As with any such scheme, usefulness is an important issue. We have explored usefulness by applying the modified WKB approximation to several problems. The examples of the spherical square well and the helium atom provide interesting numerical results. In these cases we chose a simple seed $F$ and found that the initial (variational) estimates of the ground-state energy were incorrect by several tens of percent. The first nontrivial modified WKB correction removed three-quarters of the error, or more. Where we calculated the second nontrivial modified WKB correction, the improvement was compounded. For the case of the spherical square well, the variational energy $\mathcal{E}_{2}$ was in error by $0.31 \%$, starting with $F_{4}$, which is manifestly inaccurate. ( $F_{4}$ has incorrect asymptotic behavior and a threshold potential strength only slightly below the strength used in the computation.)

In practice, the WKB method is difficult to apply to problems with many degrees of freedom. The modified WKB method does suffer from this fault. With a quadratic choice for $F$ we developed the formulas necessary for the treatment of many-body problems, and we applied them to the rather different problems of $\phi^{4}$ field theory and the helium atom.

\section{ACKNOWLEDGMENT}

This work was supported in part by the National Science Foundation under Grant Number NSF-PHY-91-21039.
[5] L. D. Landau and E. M. Lifschitz, Ref. [3], pp. 58-60.

[6] L. D. Landau and E. M. Lifschitz, Ref. [3], p. 109.

[7] L. D. Landau and E. M. Lifschitz, Ref. [3], pp. 54 and 55.

[8] S.-J. Chang, Phys. Rev. D 10, 2778 (1976).

[9] H. Bateman, Higher Transcendental Functions (McGraw-Hill, New York, 1955), Vol. 2, p. 194.

[10] L. D. Landau and E. M. Lifschitz, Ref. [3], pp. 239 and 240. 